\title{
On the convergence of circle packings to the Riemann map
}

\author{
Zheng-Xu He ${ }^{1}$, Oded Schramm ${ }^{2}$ \\ ${ }^{1}$ University of California, San Diego, USA \\ 2 The Weizmann Institute, Department of Mathematics, Rehovot 76100, Israel \\ Oblatum 15-V-1995 \& 13-XI-1995
}

\begin{abstract}
Rodin and Sullivan (1987) proved Thurston's conjecture that a scheme based on the Circle Packing Theorem converges to the Riemann mapping, thereby providing a refreshing geometric view of Riemann's Mapping Theorem. We now present a new proof of the Rodin-Sullivan theorem. This proof is based on the argument principle, and has the following virtues.

1. It applies to more general packings. The Rodin-Sullivan paper deals with packings based on the hexagonal combinatorics. Later, quantitative estimates were found, which also worked for bounded valence packings. Here, the bounded valence assumption is unnecessary and irrelevant.

2. Our method is rather elementary, and accessible to non-experts. In particular, quasiconformal maps are not needed. Consequently, this gives an independent proof of Riemann's Conformal Mapping Theorem. (The Rodin-Sullivan proof uses results that rely on Riemann's Mapping Theorem.)

3. Our approach gives the convergence of the first and second derivatives, without significant additional difficulties. While previous work has established the convergence of the first two derivatives for bounded valence packings, now the bounded valence assumption is unnecessary.
\end{abstract}

\section{Introduction}

In the following, a disk packing is a collection of closed geometric disks, with disjoint interiors, in the plane $\mathbb{C}$, or the Riemann sphere $\hat{\mathbb{C}}=\mathbb{C} \cup\{\infty\}$. An interstice of a disk packing $P$ is a connected component of the complement of $P$, and an interstice whose closure intersects only three disks in the packing is

This work was supported by NSF grant DMS94-03548. The first author was supported by an NSF grant and a Sloan Research Fellowship. The second author would like to thank MSRI for its hospitality and support. 
a triangular interstice. The support, $\operatorname{supp}(P)$, of $P$ is defined as the union of the disks in $P$ and all bounded interstices of $P$.

Let $P$ and $\widetilde{P}$ be two finite disk packings in $\mathbb{C}$. If there is an orientation preserving homeomorphism $h: \operatorname{supp}(P) \rightarrow \operatorname{supp}(\widetilde{P})$ such that $h(P)=h(\widetilde{P})$, then we shall say that $P$ and $\widetilde{P}$ are isomorphic, and $h$ will be called an isomorphism between them. It is clear that an isomorphism $h$ induces a bijection between the disks of $P$ and the disks of $\widetilde{P}$. An isomorphism of packings is essentially a combinatorial notion. One can determine if two packings are isomorphic, by looking at the pattern of tangencies of the disks, etc.

It will be notationally convenient to work with indexed packings: an indexed disk packing $P=\left(P_{v}: v \in V\right)$ is just a packing in which the disks are indexed by some set $V$. The nerve, or tangency graph, of an indexed packing $P=$ $\left(P_{v}: v \in V\right)$ is the graph on the vertex set $V$ in which $[u, v]$ is an edge iff $P_{v}$ and $P_{u}$ intersect (tangentially).

Of fundamental importance is the circle packing theorem (P. Koebe [12]), which asserts that for any finite planar graph $G=(V, E)$ there is a disk packing $P=\left(P_{v}: v \in V\right)$ in $\mathbb{C}$ whose tangency graph is $G$. Moreover, when $G$ is the 1-skeleton of a triangulation of $S^{2}$, the packing $P$ is unique up to Möbius transformations. Some later proofs of the circle packing theorem include $[4,13,2,20]$.

Now suppose that $P$ is a finite packing in $\mathbb{C}$. The disks of $P$ that intersect the boundary of the support of $P$ will be called boundary disks. It is a consequence of the circle packing theorem that there is an isomorphic disk packing $\widetilde{P}$, which is contained in the closed unit disk $U$, such that the boundary disks of $\widetilde{P}$ are all internally tangent to the unit circle $\partial U$.

Let $D$ be some bounded simply connected domain in $\mathbb{C}$, and $p_{0}$ some interior point of $D$. For each $n$, let $P^{n}$ be a disk packing in $D$, in which all bounded interstices are triangular. Assume that there is a sequence of positive numbers $\delta_{n}$ with limit 0 such that: i) the radius of any disk in the packing $P^{n}$ is at most $\delta_{n}$, and ii) any boundary disk in the packing $P^{n}$ is within $\delta_{n}$ from the boundary of $D$. Denote by $P_{0}^{n}$ a selected disk of the packing $P^{n}$ which (contains or) is closest to $p_{0}$.

As mentioned above, the circle packing theorem implies that there is an isomorphic packing $\widetilde{P}^{n}$ in the closed unit disk, with boundary disks all tangent to the unit circle $\partial U$. Let $f_{n}: \operatorname{supp}\left(P^{n}\right) \rightarrow \operatorname{supp}\left(\widetilde{P}^{n}\right)$ be an isomorphism of $P^{n}$ and $\widetilde{P}^{n}$. Normalize $\widetilde{P}^{n}$ by Möbius transformations preserving $U$ so that the disk $\widetilde{P}_{0}^{n}$ of $\widetilde{\widetilde{P}}^{n}$ corresponding to $P_{0}^{n}$ is centered at 0 .

In his talk [23], W. Thurston conjectured that the sequence of functions $f_{n}$ converges to the Riemann mapping from $D$ to $U$, if the packings $P^{n}$ are taken as subpackings of scaled copies of the infinite hexagonal circle packing. The conjecture was later proved by Rodin and Sullivan [15].

Here, a new proof is given to this result, which avoids restrictions on the combinatorics of $P^{n}$. Before this is explained, we give a sketch of the methods used by Rodin and Sullivan, and briefly describe some more recent developments. 
Rodin and Sullivan proved a local compactness result for bounded valence packings, which is known as the Ring Lemma. It shows that the maps $f_{n}$ may be taken as $K$-quasiconformal, for some fixed $K$. Hence some subsequence of $f_{n}$ converges to a limit $f$. They also prove the rigidity of the infinite hexagonal circle packing, and conclude that $f$ must be 1-quasiconformal, hence conformal. Their proof of the rigidity also works for circle packings of bounded valence whose support is the whole plane ${ }^{1}$, and it follows from their argument that the convergence to the Riemann mapping holds for non-hexagonal circle packings as well, but with the restriction that the ratio of the maximal radius and the minimal radius of the circles in each "source" packing $P_{n}$ is uniformly bounded. (The uniform bound on the ratios, rather than on the valence, is needed to guarantee that the support of a limit of rescaled circle packings is the whole plane. Rigidity fails for any circle packing whose support is a simply connected proper subdomain of $\mathbb{C}[11]$.) An analogous convergence result was proved by K. Stephenson [22], by considering electric networks and random walks on the tangency graph.

It is now time to state our generalization of the Rodin-Sullivan theorem.

1.1 Theorem Let $D, \widetilde{D} \varsubsetneqq \mathbb{C}$ be two simply connected domains, and let $p_{0}$ be some point in $D$. For each $n$, let $P^{n}$ be a disk packing in $D$, with all bounded interstices triangular, let $\widetilde{P}^{n}$ be an isomorphic packing in $\widetilde{D}$, and let $f_{n}: \operatorname{supp}\left(P^{n}\right) \rightarrow \operatorname{supp}\left(\widetilde{P}^{n}\right)$ be an isomorphism.

Let $\delta_{n}$ be a sequence of positive numbers, tending to zero, and assume that the spherical diameters ${ }^{2}$ of the disks in every $P^{n}$ are less than $\delta_{n}$, and for each boundary disk $P_{v}^{n}$ of $P^{n}$ the spherical distance from it to $\partial D$ and the spherical distance from $\partial \widetilde{D}$ to the corresponding disk $f_{n}\left(P_{v}^{n}\right)$ are less than $\delta_{n}$. Suppose that for each $n$ the point $p_{0}$ is contained in the support of $P^{n}$, and that the set $\left\{f_{n}\left(p_{0}\right): n=1,2, \ldots\right\}$ has compact closure in $\widetilde{D}$. Then a subsequence of the sequence $f_{n}$ converges uniformly on compact subsets of $D$ to a conformal homeomorphism $f: D \rightarrow \widetilde{D}$. See Figure 1.1.

Remarks. The reason for taking $\widetilde{D}$ to be an arbitrary simply connected proper subdomain of $\mathbb{C}$, rather than $\widetilde{D}=U$, is that we want the situation to be close to symmetric. There are only two assumptions that are non-symmetric: we do not yet know that the spherical diameters of the disks in $\widetilde{P}_{n}$ are tending to zero, and that there is some point $\widetilde{p}_{0} \in \widetilde{D}$ such that the set $\left\{f_{n}^{-1}\left(\widetilde{p}_{0}\right): n=1,2, \ldots\right\}$ is compact. Once we prove these statements, for any statement proven for $f_{n}$ the symmetric statement will hold for $f_{n}^{-1}$.

\footnotetext{
1 This rigidity was subsequently generalized to unbounded valence packings [19], and to circle domains [9].

${ }^{2}$ The inversion in the sphere of radius 2 about $(0,0,2)$ in $\mathbb{R}^{3}$ takes the plane $\mathbb{C}$, which we identify with $\left\{(x, y, z) \in \mathbb{R}^{3}: z=0\right\}$, to the sphere of radius 1 about the point $(0,0,1)$. The spherical metric of $\mathbb{C}$ is just the pullback of the intrinsic metric of the sphere $\left\{p \in \mathbb{R}^{3}:\|p-(0,0,1)\|=1\right\}$ under this inversion.
} 

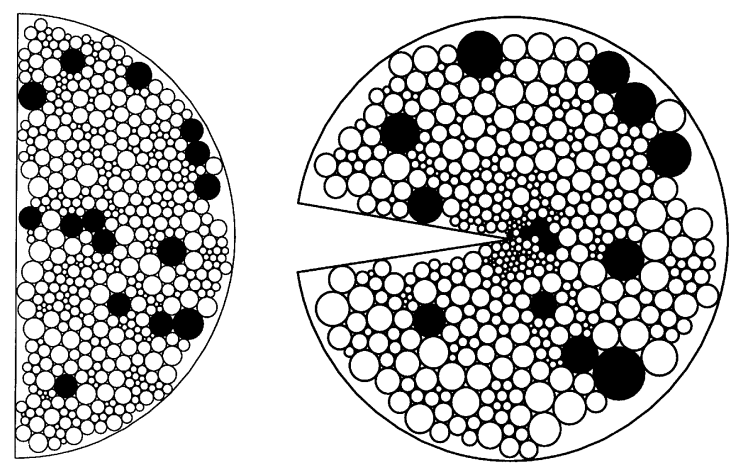

Fig. 1.1. The two packings. (Some circles are marked in both packings to indicate the correspondance.)

Given the packings $P^{n}$ in $D$, there exist isomorphic packings $\widetilde{P}^{n}$ in $\widetilde{D}$ that satisfy the assumptions of the theorem. To see this, take a sequence of Jordan domains $\widetilde{D}^{n}$ approximating $\widetilde{D}$ from the inside. By [17] (see also [18], or [20]), there is a disk packing $\widetilde{P}^{n}$ in the closure of $\widetilde{D}^{n}$ that is isomorphic to $P^{n}$ and with all boundary disks tangent to $\partial \widetilde{D}^{n}$, and one can normalize so that $f_{n}\left(p_{0}\right)$ is any specified point of $\widetilde{D}^{n}$.

The theorem claims the convergence of a subsequence, rather than the convergence of the sequence $f_{n}$. It is easy to see that this is not a significant limitation; the sequence $f_{n}$ would converge if one assumes an appropriate normalization.

We have taken the packing $\widetilde{P}^{n}$ to be contained in $\widetilde{D}$. This disallows the possibility that there are any disks tangent to $\partial \widetilde{D}$. If $\widetilde{D}$ is a Jordan domain, we could just replace the assumption $\widetilde{P}^{n} \subset \widetilde{D}$ by $\widetilde{P}^{n} \subset \widetilde{D}$. Otherwise, one can just require that the interior of the support of $\widetilde{P}^{n}$ be contained in $\widetilde{D}$. A similar remark holds for $P^{n}$ and $D$.

In a future paper, we will study the rate of convergence of the maps $f_{n}$ to the conformal map, and will prove a bound of the form $\left|f_{n}-f\right|=O\left(\delta_{n}^{\alpha}\right)$, for some exponent $\alpha>0$.

Convergence of the derivatives In [7], the first named author proved the convergence of the first derivatives of $f_{n}$ in the hexagonal case. This work was extended in [8] to the more general case when the packings $P_{n}$ have uniformly bounded valence. Actually, under the bounded valence assumption, and with similar methods, it is also possible to show that the second order derivatives exist in some sense, and they converge to the corresponding derivatives of the Riemann mapping. The proof in the hexagonal case appears in [5].

Here, we shall also prove the convergence of the first two derivatives, without the bounded valence assumption. The first step is to explain what this means. Suppose that $P$ and $\widetilde{P}$ are isomorphic disk packing, and let $h$ be an isomorphism between them. Here, and below, we assume for convenience that $P$ and $\widetilde{P}$ are indexed by the same set, $V$, and that the isomorphism between 
them respects the indexing; that is, $P=\left(P_{v}: v \in V\right), \widetilde{P}=\left(\widetilde{P}_{v}: v \in V\right)$, and $h\left(P_{v}\right)=\widetilde{P}_{v}$. Let $J$ be a triangular interstice of $P$, and let $P_{u}, P_{v}, P_{w}$ be the three disks of $P$ that intersect the closure of $J$. Then the three tangency points around $J$ are $P_{u} \cap P_{v}, P_{v} \cap P_{w}$, and $P_{w} \cap P_{u}$. Let $m_{J}$ be the Möbius transformation that satisfies $m_{J}\left(P_{u} \cap P_{v}\right)=\widetilde{P}_{u} \cap \widetilde{P}_{v}, m_{J}\left(P_{v} \cap P_{w}\right)=\widetilde{P}_{v} \cap \widetilde{P}_{w}$, and $m_{J}\left(P_{w} \cap P_{u}\right)=\widetilde{P}_{w} \cap \widetilde{P}_{u}$. Then it follows that $m_{J}\left(P_{u}\right)=\widetilde{P}_{u}, m_{J}\left(P_{v}\right)=\widetilde{P}_{v}$, and $m_{J}\left(P_{w}\right)=\widetilde{P}_{w}$, and $m_{J}$ maps $J$ onto the triangular interstice of $\widetilde{P}$ bounded by $\widetilde{P}_{u} \cup \widetilde{P}_{v} \cup \widetilde{P}_{w}$. (The last statement relies on the assumption that the isomorphism between $P$ and $\widetilde{P}$ preserves orientations.)

Let $I$ be the union of all the triangular interstices of $P$, and let $\widetilde{I}$ be the union of all triangular interstices of $\widetilde{P}$. Let $g: I \rightarrow \widetilde{I}$ be the map that agrees with $m_{J}$ in the closure of every triangular interstice $J$. Then $g$ is well defined and continuous, and is actually conformal in $I$. This map $g$ will be called the interstitial map induced by the isomorphism from $P$ to $\widetilde{P}$. It is not hard to see that the interstitial map can be extended to an isomorphism of $P$ and $\widetilde{P}$.

1.2 Theorem Let the situation be as in theorem 1.1, and assume that $f_{n}$ converges to $f$ (rather than a subsequence, for simplicity of notation). Denote the union of the bounded interstices of $P^{n}$ by $I^{n}$, and let $g_{n}$ be the induced interstitial map. Then the restrictions of $g_{n}^{\prime}$ and $g_{n}^{\prime \prime}$ to $I^{n}$ converge uniformly on compacts of $D$ to $f^{\prime}$ and $f^{\prime \prime}$, respectively; that is, given any compact $K \subset D$ and $\varepsilon>0$ there is an $N$ such that $\left|f^{\prime}(z)-g_{n}^{\prime}(z)\right|<\varepsilon$ and $\left|f^{\prime \prime}(z)-g_{n}^{\prime \prime}(z)\right|<\varepsilon$ hold for any $n>N$ and $z \in K \cap I^{n}$.

A consequence of the theorem is that the ratio diameter $\left(\widetilde{P}_{v}^{n}\right) / \operatorname{diameter}\left(P_{v}^{n}\right)$ approximates $\left|f^{\prime}(z)\right|$, when $n$ is large and $z$ is a point in $P_{v}^{n}$.

With general combinatorics, it is unclear if one can even give a reasonable definition for the third derivative of a correspondence between circle packings. On the other hand, it is not hard to come up with a good definition in the case of packings based on the hexagonal combinatorics, say. We cannot prove the convergence of the third order derivatives.

It is noteworthy that the proof of 1.2 does not require much beyond the work we do not prove 1.1. All in all, the methods we use are very elementary, and do not rely on any heavy machinery. In particular, there is no mention of quasiconformal maps, which have been so central in most works in this vein.

The first part of the proofs is in proving topological results about the sequence of maps $f_{n}$, which is done in Sect. 2. For example, the first result we shall prove is that for any compact $\widetilde{K} \subset \widetilde{D}$ the inverse images $f_{n}^{-1}(\widetilde{K})$ are all contained in one compact subset of $D$. In other words, the maps $f_{n}$ are uniformly proper. The topological results use methods that are based on the notion of extremal length, though this notion is not mentioned explicitly. Already the Rodin-Sullivan paper contains an argument of this flavor (their length-area lemma). Although the tricks we use here are somewhat more involved, they are still completely elementary. 
In Sect. 3, we recall a Lemma of Carleman [3], and derive consequences. This Lemma says that for an orientation preserving homeomorphism $g$ between circles in $\mathbb{C}$ the winding number of $g(z)-z$ around zero is nonnegative, provided $g(z)-z$ does not have zeroes. This is the circle packing analogue of the fact that the winding number of $h(z)-z$ around 0 is nonnegative, when $h$ is the restriction of an analytic function $H$ to a curve $\gamma$, which is null homotopic in the domain of $H$.

In Sect. 4, we shall see that geometric control of the interstitial maps $g_{n}$ follows from the previous discussion, and finally, in Sect. 5, the proofs of 1.1 and 1.2 will be completed. Sect. 6 contains generalizations of Theorems 1.1 and 1.2. In particular, Theorem 6.1 considers packings with non-triangular interstices.

\section{Topological behavior}

In this section, we shall assume the hypothesis and notations of Theorem 1.1.

This section, though elementary, is somewhat technical and a bit involved. The reader is advised to read the statements of the lemmas here, but return to their proofs only after reading the later sections. One may note that the results of this section, in contrast to the rest, are much simpler in the hexagonal setting. For the hexagonal setting, there is very little needed beyond the corresponding arguments of [15].

In this section, the spherical metric shall be extensively used. Metric quantities relating to the spherical metric will be marked with a subscript ${ }_{s p}$, for example, area $_{s p}$, diameter dia $_{s p}$

2.1 Lemma The maps $f_{n}$ are eventually uniformly proper onto $\widetilde{D}$; that is, given any compact $\widetilde{K} \subset \widetilde{D}$ there is a compact $K \subset D$ such that $f_{n}^{-1}(\widetilde{K}) \subset K$ for every sufficiently large $n$.

The basic idea in the proof is to control the diameter of a union of circles by estimating the area. This idea goes back to the Length-Area Lemma of [15], and is analogous to standard methods in the theory of quasiconformal maps.

Proof. Let $\widetilde{K}$ be any compact connected subset of $\widetilde{D}$ that contains the set $\left\{f_{n}\left(p_{0}\right): n=1,2, \ldots\right\}$. It is clearly enough to prove the lemma for such $\widetilde{K}$. Let $\varepsilon=d_{s p}(\widetilde{K}, \partial \widetilde{D})$.

Set $r_{1}=\min \left\{d_{s p}\left(p_{0}, \partial D\right)\right.$, diameter $\left.s p(\partial D)\right\} / 3$. Note that $\operatorname{diameter}_{s p}(\hat{\mathbb{C}})=\pi$, so $r_{1} \leqq \pi / 3$. Let $r_{0} \in\left(0, r_{1} / 2\right)$ be very small in comparison to $r_{1}$ and $\varepsilon$; just how small shall be determine below. (But $r_{0}$ will only depend on $r_{1}$ and $\varepsilon$.) Let $n$ be sufficiently large so that $\delta_{n}<\min \left\{r_{0}, \varepsilon\right\} / 5$, and let $K=\left\{z \in D: d_{s p}(z, \partial D) \geqq r_{0} / 2\right\}$. The lemma will be established by showing that $f_{n}^{-1}(\widetilde{K}) \subset K$. 
Let $z_{0}$ be some point in $\operatorname{supp}\left(P^{n}\right)-K$, then $d_{s p}\left(z_{0}, \partial D\right)<r_{0} / 2$. We need to show that $f_{n}\left(z_{0}\right) \notin \widetilde{K}$.

For $\rho>0$ let $c_{s p}(\rho)$ be the circle $c_{s p}(\rho)=\left\{z: d_{s p}\left(z, z_{0}\right)=\rho\right\}$, and let $V(\rho)$ be the set of $v \in V^{n}$ such that $P_{v}^{n}$ intersects $c_{s p}(\rho)$. Set

$$
L(\rho)=\sum_{v \in V(\rho)} \operatorname{diameter}_{s p}\left(\widetilde{P}_{v}^{n}\right) .
$$

We shall later show that

$$
\inf \left\{L(\rho): \rho \in\left[r_{0}, r_{1}\right]\right\}<\varepsilon / 2 .
$$

Assume this for the moment, and fix a $\rho \in\left[r_{0}, r_{1}\right]$ satisfying $L(\rho)<\varepsilon / 2$.

Since $d_{s p}\left(z_{0}, \partial D\right) \leqq r_{0} / 2<r_{1}$ and $d_{s p}\left(\partial D, p_{0}\right) \geqq 3 r_{1}$, we have $d_{s p}\left(z_{0}, p_{0}\right) \geqq$ $2 r_{1}$. Hence the circle $c_{s p}(\rho)$ separates $z_{0}$ from $p_{0}$. Since $\operatorname{diameter}_{s p}\left(P_{v}^{n}\right)$ $\leqq \delta_{n}<r_{0} / 2<r_{1}$ for $v \in V^{n}$, it follows that the union

$$
\bigcup_{v \in V(\rho)} P_{v}^{n} \cup\left(\hat{\mathbb{C}}-\operatorname{supp}\left(P^{n}\right)\right)
$$

separates $z_{0}$ from $p_{0}$. (Indeed, it is easy to see that there is a homotopy in $\left\{z: d_{s p}\left(z, c_{s p}(\rho)\right) \leqq \delta_{n}\right\}$ from $c_{s p}(\rho)$ to a path in that union, because whenever $J$ is an interstice of $P$ that intersects $c_{s p}(\rho)$ the two disks at the endpoints of $J \cap c_{s p}(\rho)$ must be tangent.) Set

$$
\widetilde{P}^{n}(\rho)=\bigcup_{v \in V(\rho)} \widetilde{P}_{v}^{n}
$$

From the above it follows that the union $\widetilde{P}^{n}(\rho) \cup \partial \operatorname{supp}\left(\widetilde{P}^{n}\right)$ separates $f_{n}\left(z_{0}\right)$ from $f_{n}\left(p_{0}\right)$. See Figure 2.1.

Because $d_{s p}\left(z_{0}, \partial D\right)<\rho$ and $\operatorname{diameter}_{s p}(\partial D) \geqq 2 r_{1} \geqq 2 \rho$, the circle $c_{s p}(\rho)$ must intersect $\partial D$. Therefore, the union (2.3) is connected, and the same would be true for $\widetilde{P}^{n}(\rho) \cup \partial \operatorname{supp}\left(\widetilde{P}^{n}\right)$. But every connected component of $\widetilde{P}^{n}(\rho)$ has spherical diameter at most $L(\rho)<\varepsilon / 2$ and contains boundary circles of $\widetilde{P}^{n}$, and hence $\widetilde{P}^{n}(\rho)$ is contained in the spherical $\delta_{n}+\varepsilon / 2$ neighborhood of $\partial \widetilde{D}$. Since $\delta_{n}+\varepsilon / 2<\varepsilon, \widetilde{K}$ does not intersect this latter set, and therefore is disjoint from $\widetilde{P}^{n}(\rho)$.
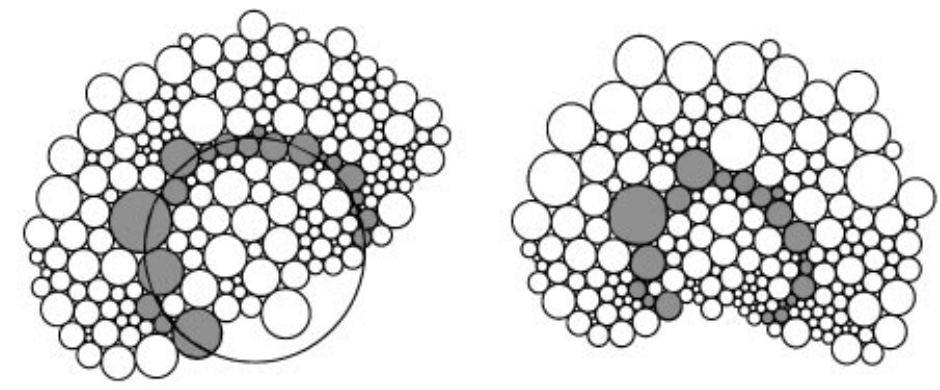

Fig. 2.1. The union $\cup_{v \in V(\rho)} P_{v}^{n}$ and the corresponding union $\widetilde{P}^{n}(\rho)$. 
We are trying to prove that $f_{n}\left(z_{0}\right) \notin \widetilde{K}$. Let $\widetilde{K}^{\prime}$ be the connected component of $\widetilde{K} \cap \operatorname{supp}\left(\widetilde{P}^{n}\right)$ that contains $f_{n}\left(p_{0}\right)$. Clearly, $f_{n}\left(z_{0}\right) \notin \widetilde{K}^{\prime}$, because $\widetilde{K}^{\prime}$ does not intersect $\widetilde{P}^{n}(\rho)$, which separates $f_{n}\left(z_{0}\right)$ from $f_{n}\left(p_{0}\right)$ in $\operatorname{supp}\left(\widetilde{P}^{n}\right)$. If $z_{1}$ is any point in $\partial \operatorname{supp}\left(P^{n}\right)$, then $d_{s p}\left(z_{1}, \partial D\right) \leqq 2 \delta_{n} \leqq r_{0} / 2$. Hence the same argument as for $z_{0}$ would apply to $z_{1}$, and show that $f_{n}\left(z_{1}\right) \notin \widetilde{K}^{\prime}$. This implies that $\widetilde{K}^{\prime} \cap \partial \operatorname{supp}\left(\widetilde{P}^{n}\right)=\emptyset$, so $\widetilde{K}^{\prime}=\widetilde{K}$ (because $\widetilde{K}$ is connected), and $f_{n}\left(z_{0}\right) \notin \widetilde{K}$. Therefore, the proof will be complete once we establish (2.2).

Let $V^{*}$ be the union of $V(\rho)$ as $\rho$ ranges in the interval $\left[r_{0}, r_{1}\right]$. This is just the set of all $v \in V^{n}$ such that $P_{v}^{n}$ intersects the annulus

$$
A=\left\{z: r_{0} \leqq d_{s p}\left(z, z_{0}\right) \leqq r_{1}\right\} .
$$

For each $v \in V^{*}$ let $\left[a_{v}, b_{v}\right]$ be the subinterval of $\left[r_{0}, r_{1}\right]$ where $v \in V(\rho)$. With these notations, we may write,

$$
\begin{aligned}
\inf & \left\{L(\rho): \rho \in\left[r_{0}, r_{1}\right]\right\} \log \left(r_{1} / r_{0}\right) \leqq \int_{r_{0}}^{r_{1}} \frac{L(\rho)}{\rho} d \rho \\
& =\sum_{v \in V^{*}} \operatorname{diameter}_{s p}\left(\widetilde{P}_{v}^{n}\right) \int_{a_{v}}^{b_{v}} \rho^{-1} d \rho \\
& \leqq \sum_{v \in V^{*}} \operatorname{diameter}_{s p}\left(\widetilde{P}_{v}^{n}\right)\left(b_{v}-a_{v}\right) / a_{v} \\
& \leqq\left(\sum_{v \in V^{*}} \operatorname{diameter}_{s p}\left(\widetilde{P}_{v}^{n}\right)^{2}\right)^{1 / 2}\left(\sum_{v \in V^{*}} \frac{\left(b_{v}-a_{v}\right)^{2}}{a_{v}^{2}}\right)^{1 / 2} .
\end{aligned}
$$

The last inequality is an application of Cauchy-Schwarz, of course. Note that there is a constant $C_{0}$ such that any disk $B \subset \hat{\mathbb{C}}$ satisfies $\operatorname{diameter}_{s p}(B)^{2} \leqq$ $C_{0} \operatorname{area}_{s p}(B)$. Since $\operatorname{area}_{s p}(\hat{\mathbb{C}})=4 \pi$ and $\widetilde{P}^{n}$ is a packing, we have

$$
\sum_{v \in V^{*}} \operatorname{diameter}_{s p}\left(\widetilde{P}_{v}^{n}\right)^{2} \leqq 4 \pi C_{0}
$$

Therefore, (2.4) gives

$$
\inf \left\{L(\rho): \rho \in\left[r_{0}, r_{1}\right]\right\} \log \left(r_{1} / r_{0}\right) \leqq 2 \sqrt{ } \pi C_{0}\left(\sum_{v \in V^{*}} \frac{\left(b_{v}-a_{v}\right)^{2}}{a_{v}^{2}}\right)^{1 / 2} .
$$

We now estimate the last sum. Note that for each $v \in V^{*}$ the disk $P_{v}^{n}$ contains a possibly smaller disk $Q_{v}$, of spherical diameter $b_{v}-a_{v}$, whose spherical distance from $z_{0}$ is $a_{v}$, and which is contained in $A$. (This latter disk will be equal to $P_{v}^{n}$, unless $a_{v}=r_{0}$ or $b_{v}=r_{1}$.) Consequently,

$$
\begin{array}{rl}
\int_{z \in A} & d\left(z_{0}, z\right)^{-2} d \text { area }_{s p} \geqq \sum_{v \in V^{*}} \int_{Q_{v}} d\left(z_{0}, z\right)^{-2} d \operatorname{area}_{s p} \\
& \geqq \sum_{v \in V^{*}} \operatorname{area}_{s p}\left(Q_{v}\right) / b_{v}^{2} \geqq \sum_{v \in V^{*}} C_{0}^{-1} \operatorname{diameter}_{s p}\left(Q_{v}\right)^{2} / b_{v}^{2} \\
& =\sum_{v \in V^{*}} C_{0}^{-1}\left(b_{v}-a_{v}\right)^{2} / b_{v}^{2} .
\end{array}
$$


Because $b_{v}-a_{v}=\operatorname{diameter}_{s p}\left(Q_{v}\right) \leqq \delta_{n}$ and $a_{v} \geqq r_{0} \geqq \delta_{n}$, we have $b_{v} \leqq 2 a_{v}$. So (2.6) gives

$$
\int_{z \in A} d_{s p}\left(z_{0}, z\right)^{-2} d \operatorname{area}_{s p} \geqq C_{0}^{-1} \frac{1}{4} \sum_{v \in V^{*}}\left(b_{v}-a_{v}\right)^{2} / a_{v}^{2} .
$$

Hence, estimating the left hand side will provide us with an estimate for the right hand side of (2.5). Recall that the spherical area of a disk of spherical radius $r$ is equal to $2 \pi(1-\cos r)$. This makes it easy to estimate the left hand side of (2.7):

$$
\begin{aligned}
& \int_{z \in A} d_{s p}\left(z_{0}, z\right)^{-2} d \operatorname{area}_{s p}=2 \pi \int_{r_{0}}^{r_{1}} t^{-2}(-d \cos t) \\
&=2 \pi \int_{r_{0}}^{r_{1}} \sin t / t^{2} d t \leqq 2 \pi \int_{r_{0}}^{r_{1}} t^{-1} d t=2 \pi \log \left(r_{1} / r_{0}\right) .
\end{aligned}
$$

Combining (2.5), (2.7) and (2.8) gives

$$
\inf \left\{L(\rho): \rho \in\left[r_{0}, r_{1}\right]\right\} \leqq 4 \sqrt{ } 2 \pi C_{0} \log \left(r_{1} / r_{0}\right)^{-1 / 2},
$$

which clearly insures (2.2), if $r_{0}$ was chosen sufficiently small. Hence the proof is complete.

2.2 Lemma Let $\widetilde{\delta}_{n}$ denote the maximal spherical diameter of any disk in $\widetilde{P}^{n}$. Then $\widetilde{\delta}_{n} \rightarrow 0$ as $n \rightarrow \infty$.

Proof. The proof will use little more than the arguments in the proof of Lemma 2.1.

Let $\varepsilon>0$. We shall establish the lemma by showing that $\operatorname{diameter}_{s p}\left(\widetilde{P}_{v}^{n}\right) \leqq$ $\varepsilon$ for all sufficiently large $n$ and $v \in V^{n}$.

Let $\widetilde{K}$ be a compact subset of $\widetilde{D}$ that contains $\left\{z \in \widetilde{D}: d_{s p}(z, \partial \widetilde{D}) \geqq \varepsilon / 2\right\}$. From Lemma 2.1 we know that there is a compact $K \subset D$ such that $f_{n}^{-1}(\widetilde{K}) \subset$ $K$ for all sufficiently large $n$.

Let $n$ be very large, and let $v \in V^{n}$. If $P_{v}^{n}$ does not intersect $K$, then it follows that $\widetilde{P}_{v}^{n}$ does not intersect $\widetilde{K}$. But the spherical distance from the (spherical) center of $\widetilde{P}_{v}^{n}$ to $\partial \widetilde{D}$ is at least $\operatorname{diameter}_{s p}\left(\widetilde{P}_{v}^{n}\right) / 2$. Since the center is not in $\widetilde{K}$, we get $\operatorname{diameter}_{s p}\left(\widetilde{P}_{v}^{n}\right)<\varepsilon$. Hence we should only consider the case where $P_{v}^{n}$ intersects $K$.

Set $\varepsilon^{\prime}=\min \left\{\varepsilon, \operatorname{diameter}_{s p}(\partial \widetilde{D})\right\}$, let $r_{1}=d_{s p}(K, \partial D) / 3$, and let $r_{0} \in$ $\left(0, r_{1} / 3\right)$, be very small in comparison with $r_{1}$ and $\varepsilon^{\prime}$. Suppose that $n$ is sufficiently large so that $\delta_{n}<r_{0} / 3$, and suppose that $P_{v}^{n}$ intersects $K$. Then $d_{s p}\left(P_{v}^{n}, \partial D\right) \geqq 3 r_{1}-\delta_{n}>2 r_{1}$, and therefore $d_{s p}\left(P_{v}^{n}, \partial \operatorname{supp}\left(P^{n}\right)\right)>2 r_{1}-2 \delta_{n}>$ $r_{1}$. Let $z_{0}$ be the spherical center of $P_{v}^{n}$.

We proceed as in the proof of Lemma 2.1. For every $\rho \in\left[r_{0}, r_{1}\right]$ let $V(\rho)$ be the set of $v \in V^{n}$ such that $P_{v}^{n}$ intersects the circle $c_{s p}(\rho)=\left\{z: d_{s p}\left(z, z_{0}\right)=\rho\right\}$, and let $\widetilde{P}^{n}(\rho)$ be the union $\cup\left\{P_{v}^{n}: v \in V(\rho)\right\}$. It then follows that $\widetilde{P}^{n}(\rho)$ separates $\widetilde{P}_{v}^{n}$ from $\partial \widetilde{D}$. The arguments in the proof of Lemma 2.1 also show 
that for some $\rho \in\left[r_{0}, r_{1}\right]$, we have $\operatorname{diameter}_{s p}\left(\widetilde{P}^{n}(\rho)\right)<\varepsilon^{\prime} / 2$, provided we have chosen $r_{0}$ sufficiently small. Because $\widetilde{P}^{n}(\rho)$ separates $\widetilde{P}_{v}^{n}$ from $\partial \widetilde{D}$, and $\operatorname{diameter}_{s p}(\partial \widetilde{D}) \geqq \varepsilon^{\prime}$. This implies that $\operatorname{diameter}_{s p}\left(\widetilde{P}_{v}^{n}\right) \leqq \varepsilon^{\prime} / 2<\varepsilon$. Thus the proof of 2.2 is complete.

2.3 Corollary Let $\widetilde{K}$ be any compact subset of $\widetilde{D}$, then $\operatorname{supp}\left(\widetilde{P}^{n}\right) \supset \widetilde{K}$ for all sufficiently large $n$. Similarly, given any compact $K \subset D$, we have $\operatorname{supp}\left(P^{n}\right) \supset$ $K$ for all sufficiently large $n$.

Proof. The latter follows from the fact that every point of $\partial \operatorname{supp}\left(P^{n}\right)$ is at distance at most $2 \delta_{n}$ from $\partial D$ and $p_{0} \in \operatorname{supp}\left(P^{n}\right)$, and $\delta_{n} \rightarrow 0$. Similarly, the first statement is a consequence of $\delta_{n}, \widetilde{\delta}_{n} \rightarrow 0$ and the fact that $\operatorname{supp}\left(\widetilde{P}^{n}\right)$ contains $f_{n}\left(p_{0}\right)$, which stays in a compact subset of $\widetilde{D}$.

2.4 Corollary Given any compact $K \subset D$, there is a compact $\widetilde{K} \subset \widetilde{D}$ such that $f_{n}(K) \subset \widetilde{K}$ for all sufficiently large $n$.

Proof. Let $\widetilde{p}_{0}$ be any point in $\widetilde{D}$. Then Lemma 2.1 tells us that $\left\{f_{n}^{-1}\left(\widetilde{p}_{0}\right)\right.$ : $n>N\}$ is contained in a compact subset of $D$, if $N$ is sufficiently large. This together with Lemma 2.2 shows that we may exchange $D$ for $\widetilde{D}, \delta_{n}$ for $\delta_{n}+\widetilde{\delta}_{n}, f_{n}$ for $f_{n}^{-1}$ and $P^{n}$ for $\widetilde{P}^{n}$, and the assumptions in Theorem 1.1 are still satisfied. Then 2.4 is just an application of Lemma 2.1.

\section{The circle fixed point index lemma}

The previous section contained results giving rough control over the mappings $f_{n}$. The instrument for gaining precise geometric control is the following lemma, which is originally due to Carleman [3].

3.1 Circle fixed point index lemma Let $C_{1}, C_{2}$ be two circles in $\mathbb{C}$, positively oriented with respect to the disks they bound. Suppose that $\phi: C_{1} \rightarrow C_{2}$ is an orientation preserving homeomorphism, that has no fixed points. Then the winding number of the curve $\left\{\phi(z)-z: z \in C_{1}\right\}$ around 0 is non-negative.

To see the connection with fixed points, consider a map $\Phi$ that maps the disk bounded by $C_{1}$ into $\mathbb{C}$ and agrees with $\phi$ on $C_{1}$. Then the number of fixed points of $\Phi$, when appropriately interpreted, is equal to the winding number of $\left\{\phi(z)-z: z \in C_{1}\right\}$ around 0 .

Carleman used this lemma to prove that a conformal mapping between finitely connected circle domains is a Möbius transformation. In the paper [9], we have used it to study rigidity properties of circle domains and circle packings.

Lemma 3.1 is a consequence of the following.

3.2 Lemma Let $\gamma_{1}, \gamma_{2}$ be two Jordan curves in $\mathbb{C}$, oriented positively with respect to the disks they bound. Suppose that $\gamma_{1} \cap \gamma_{2}$ contains at most two points, 
or $\gamma_{1}=\gamma_{2}$. Let $g: \gamma_{1} \rightarrow \gamma_{2}$ be an orientation preserving homeomorphism, with no fixed points. Then the winding number of $g(z)-z$ around 0 is nonnegative.

See [3], or [9] for a proof.

Remark. The proof of 3.2 implies an even more general version: suppose that $g_{1}: \partial U \rightarrow \gamma_{1}$ and $g_{2}: \partial U \rightarrow \gamma_{2}$ are orientation preserving covering maps and $g_{1} \neq g_{2}$ on $\partial U$, then the winding number of $g_{1}-g_{2}$ around zero is nonnegative. This stronger form should be useful for considering branched packings.

Beardon and Stephenson have shown [1] that a version of the Schwarz-Pick Lemma holds for circle packings. In [9] a different version was proved, using the circle fixed point index lemma. Here we shall present a special case of the circle Schwarz-Pick Lemma, in a form most suitable to the applications that follow.

3.3 The circle Schwarz lemma Let $\Omega, \widetilde{\Omega} \subset \mathbb{C}$ be two bounded Jordan domains $^{3}$. Suppose that $\widetilde{\Omega} \subset U \subset \Omega$, where $U=\{z:|z|<1\}$ is the unit disk. Let $P$ be a finite packing of disks in the closure of $\Omega$, and let $\widetilde{P}$ be a finite packing of disks in the closure of $\widetilde{\Omega}$. Let $A=\Omega$-interior $(P)$ (the closure of $\Omega$ minus the union of the interiors of disks in $P$ ), and let $\widetilde{A}=\widetilde{\Omega}-$ interior $(\widetilde{P})$. Suppose that $f: A \rightarrow \widetilde{A}$ is a homeomorphism that is conformal in the interior of $A$ and $f(\partial \Omega)=\partial \widetilde{\Omega}$. Assume that 0 is in the interior of $A$ and $f(0)=0$. Then $\left|f^{\prime}(0)\right| \leqq 1$. See Fig. 3.1.

The proof will be a good warmup to what follows.

Note that $f\left(\partial P_{v}\right)$ is the boundary of a disk in $\widetilde{P}$, whenever $P_{v}$ is a disk in $P$, because $f\left(\partial P_{v}\right)$ is a Jordan curve that does not separate $\widetilde{A}$ and is not equal to $\partial \widetilde{\Omega}$.

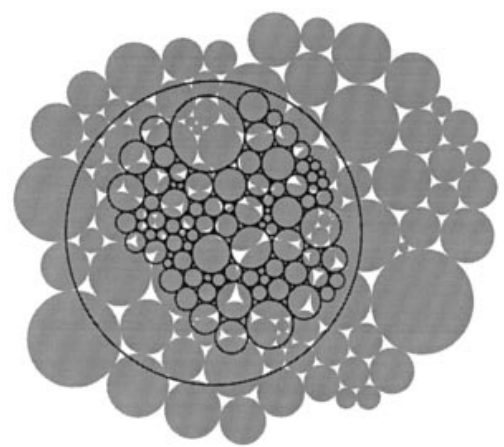

Fig. 3.1. The setup in the Circle Schwarz Lemma.

\footnotetext{
${ }^{3}$ A Jordan domain is a domain whose boundary is a Jordan curve.
} 
Proof. Set $\lambda=f^{\prime}(0)$, and suppose that $|\lambda|>1$. Set $g(z)=(f(z) / \lambda)-z$, and assume for the moment that $g$ has no zeroes in $\partial A$. Then the total number of zeroes of $g$ in $A$, counting multiplicities, is equal to the winding number around 0 of the restriction of $g$ to $\partial A$.

Consider some disk $P_{v}$ in the packing $P$. From the circle fixed point index lemma, we know that the winding number around 0 of $g$ restricted to $\partial P_{v}$ is nonnegative, if $\partial P_{v}$ is taken with the orientation as the boundary of $P_{v}$. But for computing the contribution to the winding number of $g$ restricted to $\partial A$, we should take the opposite orientation of $\partial P_{v}$. Hence the contribution is nonpositive.

The part of $\partial A$ that is not contained in the disks of $P$ is all in $\partial \Omega$. Consider the homotopy $h(z, s)=z-s f(z) / \lambda, s \in[0,1], z \in \partial \Omega$, from the identity to $-g$. Since $|f(z) / \lambda|<1$, and $|z| \geqq 1$ for $z \in \partial \Omega$, the homotopy does not have any zeroes in $\partial \Omega$. Hence the winding number of $g$ restricted to $\partial \Omega$ around zero, which is also the winding number of $-g$ restricted to $\partial \Omega$, is equal to the winding number of the identity restricted to $\partial \Omega$ around 0 , which is 1 .

From the above two paragraphs it follows that the total winding number of $g$ restricted to $\partial A$ around 0 is at most 1 . But $g(0)=0$ and $g^{\prime}(0)=\left(f^{\prime}(0) / \lambda\right)-1=$ 0 , so $g$ has a double zero at 0 . This is a contradiction, which shows that either $\left|f^{\prime}(0)\right| \leqq 1$, or $g$ has zeroes in $\partial A$.

Suppose that $g$ has zeroes in $\partial A$. Since $A$ is connected, and $g$ cannot have zeroes in $\partial \Omega$, there must be some point $p \in A$, which is a zero of $g$ and such that $g$ is not constant in a neighborhood of $p$. There must be some component of the interior of $A$ that has $p$ in its closure, and where $g$ is not constant. Therefore, if $W$ is a small open set containing $p$, then $\{g(z): z \in W \cap A\}$ contains an open set in a small neighborhood of 0 . Consequently, the set of $\lambda^{\prime}$ close to $\lambda$ such that $g_{\lambda^{\prime}}(z)=\left(f(z) / \lambda^{\prime}\right)-z$ has a zero near $p$ has interior. This implies that there will be some $\lambda^{\prime}$ with $\left|\lambda^{\prime}\right|>1$ such that $g_{\lambda^{\prime}}$ has no zeroes on $\partial A$ and has a zero near $p$. (The set of $\lambda^{\prime} \neq 0$ such that $g_{\lambda^{\prime}}$ has a zero on $\partial A$ is the image of $\partial A$ under the map $f(z) / z$. Clearly, this set has empty interior.) Then this $g_{\lambda^{\prime}}$ has two zeroes in $A$ and none in $\partial A$, which gives a contradiction, as above. Therefore, $\left|f^{\prime}(0)\right| \leqq 1$.

The following lemma is similar to the above, but involves two derivatives.

3.4 Lemma Let $\Omega, \widetilde{\Omega}$ be bounded Jordan domains in $\mathbb{C}$, let $P$ be a disk packing contained in the closure of $\Omega$, and let $\widetilde{P}$ be a disk packing contained in the closure of $\widetilde{\Omega}$. Let $A=\Omega$-interior $(P)$, and let $\widetilde{A}=\widetilde{\Omega}-$ interior $(\widetilde{P})$. Suppose that $f: A \rightarrow \widetilde{A}$ is a homeomorphism, which is conformal in the interior of $A$, and takes $\partial \Omega$ to $\partial \widetilde{\Omega}$. Let $w_{0}$ be an interior point of $A$, and let $m$ be the Möbius transformation that satisfies $m\left(w_{0}\right)=f\left(w_{0}\right), m^{\prime}\left(w_{0}\right)=f^{\prime}\left(w_{0}\right)$, and $m^{\prime \prime}\left(w_{0}\right)=f^{\prime \prime}\left(w_{0}\right)$. Then $f(\partial \Omega) \cap m(\partial \Omega) \neq \emptyset$. 
Proof. Let $\psi$ be the Möbius transformation $\psi(z)=w_{0}+\frac{1}{z-w_{0}}$. Then $\psi\left(w_{0}\right)=$ $\infty$, and $\psi \circ \psi(z)=z$. Consider the map $g: \psi(A) \rightarrow \psi\left(m^{-1}(\widetilde{A})\right)$, defined by $g=\psi \circ m^{-1} \circ f \circ \psi$. It is easy to see that $m^{-1} \circ f(z)=z+o\left(\left(z-w_{0}\right)^{2}\right)$, as $z \rightarrow w_{0}$, and therefore,

$$
\lim _{z \rightarrow \infty} g(z)-z=0
$$

Fix some $\varepsilon>0$. We want to show that the distance from $\psi(\partial \Omega)$ to $g(\psi(\partial \Omega))$ is less than $\varepsilon$. If $g(z)=z$ for some point in $\psi(\partial \Omega)$, then $f(\psi(z))=$ $m(\psi(z))$, and the lemma clearly holds. Otherwise, since $\mathbb{C}$-interior $(P)$ is connected and (3.1) holds, it follows that there is some connected component $W$ of $\Omega-P$ such that $g(z)-z$ is not constant in $\psi(W)$ and $|g(z)-z|<\varepsilon$ for some $z \in \psi(W)$. Hence there is an open nonempty set of $c$ in the disk $\{z:|z|<\varepsilon\}$, such that $g(z)-z-c$ has a zero in the interior of $\psi(A)$. Therefore, we may find a $c$ satisfying $0<|c|<\varepsilon$ such that $g(z)-z-c$ has a zero in the interior of $\psi(A)$, but no zeroes on $\psi(\partial A-\partial \Omega)$. (The image of $\psi(\partial A-\partial \Omega)$ under the map $z \rightarrow g(z)-z$ cannot cover a nonempty open set.)

Set $g_{c}(z)=g(z)-c$, and $h(z)=g_{c}(z)-z=g(z)-z-c$. Assume that the distance from $\psi(\partial \Omega)$ to $g(\psi(\partial \Omega))$ is at least $\varepsilon$. We shall see that this leads to a contradiction.

Let $B$ be a small open disk centered at $w_{0}$ that is contained in the interior of $A$, and set $A^{\prime}=A-B$. We shall look at, wind ${ }_{0}\left(\left.h\right|_{\partial \quad\left(A^{\prime}\right)}\right)$, the winding number around 0 of the restriction of $h$ to $\partial \quad\left(A^{\prime}\right)$.

Note that $\psi\left(A^{\prime}\right)$ has $\psi(\partial B)$ as its outside boundary component, and $\psi(\partial B)$ is a very large circle, with center $w_{0}$. Since $\lim _{z \rightarrow \infty} h(z)=-c$, by taking $B$ to be small we ensure that the winding number of $h$ along $\psi(\partial B)$ is zero.

The winding number of $h$ along $\partial \quad\left(P_{v}\right)$ is nonnegative, for each disk $P_{v}$ in $P$. This follows from the circle fixed point index lemma 3.1, because $g_{c}$ is orientation preserving and has no fixed points in $\partial\left(P_{v}\right)$.

Since $\partial \quad(\Omega)$ and $g_{c}(\partial(\Omega))$ are disjoint, by assumption, and $g_{c}$ maps the first into the second while preserving orientations, it follows from Lemma 3.2 that the winding number of $h$ along $\partial \quad(\Omega)$ is nonnegative, if $\partial \quad(\Omega)$ is oriented positively with respect to the domain in $\mathbb{C}$ which it bounds. But $\partial \quad(\Omega)$ has the opposite orientation, so $\operatorname{wind}_{0}\left(\left.h\right|_{\partial}(\Omega) \leqq 0\right.$.

Note that

$$
\operatorname{wind}_{0}\left(\left.h\right|_{\partial}\left(A^{\prime}\right)\right)=-\operatorname{wind}_{0}\left(\left.h\right|_{\partial \quad(B)}\right)+\operatorname{wind}_{0}\left(\left.h\right|_{\partial \quad(\Omega)}\right)-\sum \operatorname{wind}_{0}\left(\left.h\right|_{\partial}\left(P_{v}\right),\right.
$$

where the sum extends over all disks $P_{v}$ in $P$. (The minus signs arise from the reversal of orientation.) Consequently, wind $_{0}\left(\left.h\right|_{\partial}\left(A^{\prime}\right) \leqq 0\right.$. But that is impossible, since $h$ is analytic in the interior of $A^{\prime}$ and has a zero there, by the choice of $c$.

Therefore, the distance from $\partial \quad(\Omega)$ to $g(\partial \quad(\Omega))$ is at most $\varepsilon$. Because that is true for every $\varepsilon>0$, we have $\partial \quad(\Omega) \cap g(\partial \quad(\Omega)) \neq \emptyset$. The lemma follows. 


\section{Geometric behaviour}

Recall that $I^{n}$ denotes the union of the triangular interstices of $P^{n}$, and $g_{n}$ : $I^{n} \rightarrow \widetilde{I^{n}}$ denotes the interstitial map.

4.1 Lemma $g_{n}^{\prime}$ is well defined and continuous.

Proof. Suppose that $P_{v}^{n}$ and $P_{u}^{n}$ are two tangent disks in $P^{n}$, and let $q$ be their point of tangency. Let $m_{1}$ be the Möbius transformation that agrees with $g_{n}$ in one interstice whose closure contains $q$, and let $m_{2}$ be the Möbius transformation that agrees with $g_{n}$ in the other interstice. Clearly, all we need to prove is that $m_{1}^{\prime}(q)=m_{2}^{\prime}(q)$.

The Möbius transformation $m_{1}$ takes $P_{v}^{n}$ onto $\widetilde{P}_{v}^{n}$ and takes $P_{u}^{n}$ onto $\widetilde{P}_{u}^{n}$, and the same is true for $m_{2}$. Hence $m_{2}^{-1} \circ m_{1}$ takes $P_{v}^{n}$ onto itself and takes $P_{u}^{n}$ onto itself.

Now let $\phi(z)=q+\frac{1}{z-q}$. Then $\phi$ is a Möbius transformation that takes $q$ to $\infty$ and $\phi \circ \phi(z)=z$. The Möbius transformation $\phi \circ m_{2}^{-1} \circ m_{1} \circ \phi$ then takes the line $\phi\left(\partial P_{v}^{n}\right)$ into itself and the line $\phi\left(\partial P_{u}^{n}\right)$ into itself. Hence it must have the form $z \rightarrow z+b$, for some $b \in \mathbb{C}$. This means that $m_{2}^{-1} \circ m_{1}=\phi(b+\phi(z))$, and so the derivative of $m_{2}^{-1} \circ m_{1}$ at $q$ is 1 . Therefore $m_{2}^{\prime}(q)=m_{1}^{\prime}(q)$, which proves the lemma.

4.2 Lemma For any compact $K \subset D$ there is an $L>0$ such that for every sufficiently large $n$ the map $g_{n}$ is L-bilipschitz on $K \cap I^{n}$. That is,

$$
L^{-1}\left|z_{1}-z_{2}\right| \leqq\left|g_{n}\left(z_{1}\right)-g_{n}\left(z_{2}\right)\right| \leqq L\left|z_{1}-z_{2}\right|
$$

holds for all sufficiently large $n$ and all $z_{1}, z_{2} \in K \cap I^{n}$.

Proof. We suppose, with no loss of generality, that $K$ is connected. The first goal is to prove a local statement:

there is an $L_{1}$ such that $\left|g_{n}^{\prime}\right|<L_{1}$ in $K \cap I^{n}$ for every sufficiently large $n$.

Let $K^{*}$ be a compact subset of $D$ that contains $K$ in its interior, and let $r_{1}>0$ be the distance from $K$ to $\mathbb{C}-K^{*}$. From 2.4 we know that there is a compact $\widetilde{K}^{*} \subset \widetilde{D}$ such that $f_{n}\left(K^{*}\right) \subset \widetilde{K}^{*}$ holds for all sufficiently large $n$. Let $R$ be the Euclidean diameter of $\widetilde{K}^{*}$, and let $n$ be sufficiently large so that $f_{n}\left(K^{*}\right) \subset \widetilde{K}^{*}$ and any disk in $P^{n}$ that intersects $K^{*}$ has Euclidean diameter at most $r_{1} / 5$.

Let $z_{0} \in I^{n}$ satisfy $d\left(z_{0}, K\right)<r_{1} / 5$. Let $A_{0}$ be the union of all disks in $P^{n}$ that intersect the disk $\left\{z:\left|z-z_{0}\right| \leqq r_{1} / 5\right\}$, let $A_{1}$ be the union of all disks in $P^{n}$ that intersect $A_{0}$, and let $\Omega$ be the union of $A_{1}$ with the bounded components of $\mathbb{C}-A_{1}$. It is not hard to see that $\Omega$ is the closure of a Jordan domain. See Fig. 4.1. Let $P$ be the collection of disks in $P^{n}$ that are contained in $\Omega$, and let $A=\Omega$-interior $(P)$. Note that $\Omega$ contains the disk of radius $r_{1} / 5$ around $z_{0}$, and the closure of $\Omega$ is contained in $K^{*}$. Consequently, $g_{n}(A) \subset \widetilde{K}^{*}$, which 


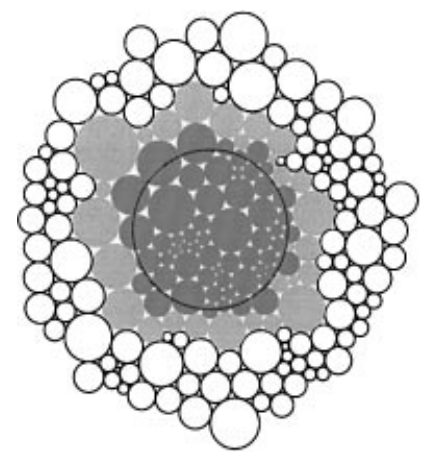

Fig. 4.1. The construction of $\Omega$. The circles in $A_{1}$ are lightly shaded, and the circles in $A_{0}$ are shaded darker.

implies that $g_{n}(A)$ is contained in a disk of radius $R$ about $g\left(z_{0}\right)$. Therefore, after appropriate translation and scaling, Lemma 3.3 gives $\left|g_{n}^{\prime}\left(z_{0}\right)\right| \leqq R /\left(r_{1} / 5\right)$.

This bound on $\left|g_{n}^{\prime}\right|$ is valid for any $z_{0}$ in $I^{n}$ intersect with the $r_{1} / 5$ neighborhood of $K$. Hence it is also valid for $I^{n} \cap K$. So (4.1) is established.

The global version $\left|g\left(z_{0}\right)-g\left(z_{1}\right)\right| \leqq L_{1}\left|z_{0}-z_{1}\right|$ in $K \cap I^{n}$ would follow easily from (4.1), if $K \cap I^{n}$ was convex. It will be helpful that $K \cap I^{n}$ is 'almost convex'. Since $K$ is connected and compact, there is some constant $C$ such that any two points $z_{0}, z_{1}$ in $K$ can be connected by a piecewise linear simple path $\gamma \subset\left\{z: d(z, K)<r_{1} / 5\right\}$ with length $(\gamma) \leqq C\left|z_{0}-z_{1}\right|$. Consider points $z_{0}, z_{1} \in K \cap I^{n}$, and let $\gamma$ be as above. For every disk $P_{v}^{n}$ in the packing $P^{n}$ that $\gamma$ intersects, we replace each component of $\gamma \cap P_{v}^{n}$ by the shortest arc along $\partial P_{v}^{n}$ that connects the two endpoints of this component. In this way, we get a new path $\gamma^{*}$, that satisfies

$$
\text { length }\left(\gamma^{*}\right) \leqq \pi \text { length }(\gamma) \leqq \pi C\left|z_{0}-z_{1}\right| .
$$

Note that $\gamma^{*}$ lies entirely in $I^{n} \cap K^{*}$. (We still assume that $n$ is large enough so that any disk in $P^{n}$ that intersects $K^{*}$ has diameter at most $r_{1} / 5$.) Since (4.1) also holds for $K^{*}$, with some constant $L_{1}=L_{1}^{*}$, we get

$$
\left|g\left(z_{0}\right)-g\left(z_{1}\right)\right| \leqq \operatorname{length}\left(\gamma^{*}\right) L_{1}^{*} \leqq \pi C L_{1}^{*}\left|z_{0}-z_{1}\right| .
$$

This proves one half of the lemma. The other is proved in the same way, by considering $g_{n}^{-1}$ in place of $g_{n}$.

4.3 Lemma Given any compact $K \subset D$ there is a constant $L$ such that $g_{n}^{\prime}$ is L-Lipschitz in $K \cap I^{n}$ for each $n$.

Proof. Clearly, it is enough to prove the lemma for $n$ sufficiently large, since each $g_{n}^{\prime}$ is $L_{n}$-Lipschitz in $K \cap I^{n}$ for some $L_{n}=L_{n}(K)$. We start with a local argument. Let $\varepsilon$ be sufficiently small so that the $\varepsilon$-neighborhood of $K$ is contained in $D$, and let $K^{\prime}$ be the closed $(\varepsilon / 2)$-neighborhood of $K$. Let $p \in K \cap I^{n}$, and let $B$ be the $(\varepsilon / 4)$-disk centered around $p$. Suppose that $n$ is 
large enough so that $K^{\prime}$ is contained in the support of $P^{n}$ and every disk of $P^{n}$ that intersects $K^{\prime}$ has diameter at most $\varepsilon / 20$.

Let $A_{0}$ be the union of all disks in $P^{n}$ that intersect $B$, let $A_{1}$ be the union of all disks in $P^{n}$ that intersect $A_{0}$, and let $\Omega$ be the union of $A_{1}$ with the bounded components of $\mathbb{C}-A_{1}$. It is easy to see that $\Omega$ is homeomorphic to a closed disk, and that $\Omega \subset K^{\prime}$. See Fig. 4.1.

Let $r$ be the distance from $p$ to $\partial \Omega$. Since $\Omega \supset B, r \geqq \varepsilon / 4$. Let $\widetilde{r}$ be the distance from $g_{n}(p)$ to the boundary of $f_{n}(\Omega)$. We now show that $\widetilde{r}$ can be bounded below by a positive quantity that depends on $K$ and $\varepsilon$, but does not depend on $n$. In fact, by 2.4 there is a compact $\widetilde{K} \subset \widetilde{D}$, independent of $n$, such that $f_{n}(\Omega) \subset f_{n}\left(K^{\prime}\right) \subset \widetilde{K}$. Applying Lemma 4.2, we conclude that

$$
\widetilde{r} \geqq \widetilde{L}^{-1} r \geqq \widetilde{L}^{-1} \varepsilon / 4,
$$

where $\widetilde{L}$ depends only on $\widetilde{K}$.

Now let $m$ be the Möbius transformation that agrees with $g_{n}$ on an interstice of $P^{n}$ containing $p$. We are looking for an upper bound on $m^{\prime \prime}(p)$. From Lemma 3.4 it follows that there is a point $q \in \partial \Omega$ such that $m(q) \in g_{n}(\partial \Omega)$. Set $\varepsilon^{\prime}=\min \left(\varepsilon / 4, \widetilde{L}^{-1} \varepsilon / 4\right)$. So we have

$$
|q-p| \geqq \varepsilon^{\prime}, \quad|m(q)-m(p)| \geqq \widetilde{r} \geqq \varepsilon^{\prime} .
$$

From Lemma 4.2 we know that there is a finite $\Lambda$, depending only on $K$, such that

$$
\left|m^{\prime}(p)\right| \leqq \Lambda .
$$

We will now show that (4.2) and (4.3) give a uniform upper bound on $\left|m^{\prime \prime}(p)\right|$. Write $m$ in the form

$$
m(z)=\frac{a z+b}{c z+d},
$$

with $a d-b c=1$. Then

$$
m^{\prime}(p)=(c p+d)^{-2},
$$

and we want to bound

$$
m^{\prime \prime}(p)=\frac{-2 c}{(c p+d)^{3}} .
$$

By (4.2),

$$
\varepsilon^{\prime} \leqq|m(q)-m(p)|=\frac{|q-p|}{|c p+d||c q+d|},
$$

which gives

$$
|c q+d| \leqq \frac{|q-p|}{|c p+d| \varepsilon^{\prime}} .
$$

Therefore,

$$
|c(q-p)| \leqq|c q+d|+|-c p-d| \leqq \frac{|q-p|}{|c p+d| \varepsilon^{\prime}}+|c p+d| .
$$


Divide by $|q-p||c p+d|^{3}$ and apply to (4.5), to get,

$$
\left|m^{\prime \prime}(p)\right| \leqq 2|c p+d|^{-4} \varepsilon^{-1}+2|q-p|^{-1}|c p+d|^{-2} .
$$

Applying (4.3), (4.4) and (4.2) here, we obtain

$$
\left|m^{\prime \prime}(p)\right| \leqq 2 \varepsilon^{\prime-1}\left(\Lambda^{2}+\Lambda\right),
$$

which is a uniform bound on $\left|m^{\prime \prime}(p)\right|$.

This bound implies that for every point $p \in K \cap I^{n}$ there is some open $W$ containing $p$ such that $g_{n}^{\prime}$ is Lipschitz with constant $1+2 \varepsilon^{\prime-1}\left(\Lambda^{2}+\Lambda\right)$ in $W \cap K \cap I^{n}$. Now an argument similar to the one given near the end of the proof of Lemma 4.2 completes the proof.

\section{Convergence to conformal maps}

We are now ready to prove our main theorem. After the work we have done, it is a breeze.

Proof (of 1.1). Let $K$ be a compact subset of $D$. From Lemmas 4.3 and 4.2 it follows easily, as in the Arzela-Ascoli Theorem [16, Th. 7.23], that a subsequence $\left\{g_{n_{j}}^{\prime}\right\}$ of the sequence $\left\{g_{n}^{\prime}\right\}$ converges uniformly in $K$, in the following sense. There is a continuous function $h: K \rightarrow \mathbb{C}$, and for every $\varepsilon>0$ there is an $N$ such that $\left|h(z)-g_{n_{j}}^{\prime}(z)\right|<\varepsilon$ holds for every $j>N$ and $z \in I^{n} \cap K$. By taking a further subsequence, if necessary, we may assume that $g_{n_{j}}^{\prime}$ converges to a continuous function $h: D \rightarrow \mathbb{C}$, uniformly on compacts in $D$, and that $f_{n_{j}}\left(p_{0}\right)$ converges.

Since $g_{n}$ is the restriction of $f_{n}$ to $I^{n}$, we know from Lemma 4.2 that $f_{n}$ is Lipschitz on $I^{n}$. Because the sizes of the disks in $\widetilde{P}^{n}$ are tending to zero as $n \rightarrow \infty$, it follows that $\left\{f_{n}\right\}$ is an equicontinuous family. Therefore, some subsequence of $f_{n_{j}}$ converges uniformly on compacts to some function $f: D \rightarrow \mathbb{C}$. Assume, without loss of generality, that this is true for the sequence $f_{n_{j}}$. We now show that $f^{\prime}=h$. In fact, let $z_{0}$ in $D$, let $\varepsilon>0$, and let $r>0$ be smaller than the distance from $z_{0}$ to $\partial D$ and such that $\left|h\left(z_{0}\right)-h(z)\right|<\varepsilon$ holds whenever $\left|z-z_{0}\right|<r$. Suppose that $z_{1} \neq z_{0}$ satisfies $\left|z_{1}-z_{0}\right|<r /(4 \pi)$ and take $n=n_{j}$ to be very large. Let $z_{1}^{\prime}$ be a closest point to $z_{1}$ that is in $I^{n}$, let $z_{2}^{\prime}$ be a closest point to $z_{2}$ that is in $I^{n}$, and let $\gamma$ be a piecewise smooth path in $I^{n}$ from $z_{0}^{\prime}$ to $z_{1}^{\prime}$ that satisfies length $(\gamma) \leqq \pi\left|z_{0}^{\prime}-z_{1}^{\prime}\right|$, as in the proof of Lemma 4.2. Then

$$
\begin{aligned}
\left|\frac{f_{n}\left(z_{1}^{\prime}\right)-f_{n}\left(z_{0}^{\prime}\right)}{z_{1}^{\prime}-z_{0}^{\prime}}-h\left(z_{0}\right)\right| & =\left|\frac{\int_{\gamma}\left(g_{n}^{\prime}(z)-h\left(z_{0}\right)\right) d z}{z_{1}^{\prime}-z_{0}^{\prime}}\right| \\
& \leqq \frac{\int_{\gamma}\left|g_{n}^{\prime}(z)-h\left(z_{0}\right)\right| d z}{\left|z_{1}^{\prime}-z_{0}^{\prime}\right|} \leqq \pi \varepsilon .
\end{aligned}
$$


When $n=n_{j} \rightarrow \infty$, while $z_{0}, z_{1}$ are kept fixed, we have $z_{0}^{\prime} \rightarrow z_{0}, z_{1}^{\prime} \rightarrow z_{1}$, $f_{n}\left(z_{0}^{\prime}\right) \rightarrow f\left(z_{0}\right)$, and $f_{n}\left(z_{1}^{\prime}\right) \rightarrow f\left(z_{1}\right)$. Therefore, we get

$$
\left|\frac{f\left(z_{1}\right)-f\left(z_{0}\right)}{z_{1}-z_{0}}-h\left(z_{0}\right)\right| \leqq \varepsilon \pi,
$$

which proves that $f^{\prime}=h$.

It only remains to show that $f$ is injective and $f(D)=\widetilde{D}$. The fact that $f(D) \subset D$ is clear, by 2.4. The argument symmetric to the above, which deals with $f_{n}^{-1}$ in place of $f_{n}$, shows that (a subsequence of) $f_{n_{j}}^{-1}$ converges uniformly on compacts in $\widetilde{D}$ to some analytic $\widetilde{f}: \widetilde{D} \rightarrow D$. By taking limits, it is then clear that $f \circ \widetilde{f}(\widetilde{z})=\widetilde{z}$ and $\widetilde{f} \circ f(z)=z$ for every $z \in D$ and $\widetilde{z} \in \widetilde{D}$. Hence $f(D)=\widetilde{D}$ and $f$ is injective. This completes the proof of the theorem.

Note that in the proof we have also demonstrated the convergence of $g_{n_{j}}^{\prime}$ to $f^{\prime}$. This will be proved again now, when we establish the convergence of the second derivative.

Proof (of 1.2). The idea of the proof is to look at the winding number around 0 of the restriction of $f_{n}-m_{n}$ to a small circle about $z$, where $z \in I^{n}$ and $m_{n}$ is the Möbius transformation agreeing with $f_{n}$ in a neighborhood of $z_{0}$.

Let $z_{0}$ be some point in $D$. Suppose that $w_{n}$ is a sequence tending to $z_{0}$ such that for all $n$ the point $w_{n}$ is in the interior of $I^{n}$. We shall show that a subsequence of $g_{n}^{\prime \prime}\left(w_{n}\right)$ converges to $f^{\prime \prime}\left(z_{0}\right)$, and a subsequence of $g_{n}^{\prime}\left(w_{n}\right)$ converges to $f^{\prime}\left(z_{0}\right)$. Clearly, this will establish the theorem.

Let $m_{n}$ be the Möbius transformation that agrees with $g_{n}$ in the interstice containing $w_{n}$. From Lemmas 2.4,4.2 and 4.3 we know that the sequences $m_{n}\left(w_{n}\right), m_{n}^{\prime}\left(w_{n}\right)$ and $m_{n}^{\prime \prime}\left(w_{n}\right)$ are bounded. Since we may pass to a subsequence, we assume, with no loss of generality, that these sequences converge. Since a Möbius transformation $m$ is determined by its value and first two derivatives at a point (when these are finite), it follows that the sequence $m_{n}$ converges to a Möbius transformation $m$, which satisfies $m^{\prime \prime}\left(z_{0}\right)=\lim _{n} m_{n}^{\prime \prime}\left(w_{n}\right)$ and $m^{\prime}\left(z_{0}\right)=$ $\lim _{n} m_{n}^{\prime}\left(w_{n}\right)$. So it suffices to prove that $m^{\prime \prime}\left(z_{0}\right)=f^{\prime \prime}\left(z_{0}\right)$ and $m^{\prime}\left(z_{0}\right)=f^{\prime}\left(z_{0}\right)$.

If $m=f$, there is nothing to prove. Otherwise, there is some small $r>0$ such that $m(z)-f(z)$ has no zeroes in the deleted disk $\left\{z: 0<\left|z-z_{0}\right| \leqq 2 r\right\}$. Assume that $r$ is sufficiently small so that the closed disk of radius $3 r$ around $z_{0}$ is contained in $D$ and $m$ has no poles in it. Let $\alpha$ be the circle $\left\{z:\left|z-z_{0}\right|=r\right\}$, and let $\gamma$ be the restriction of $m(z)-f(z)$ to $\alpha$. Then $\gamma$ is a closed path in $\mathbb{C}-\{0\}$. We shall prove that the winding number of $\gamma$ around 0 is at least 3. This will show that there are at least three zeroes of $m-f$ in the disk $\left\{z:\left|z-z_{0}\right| \leqq r\right\}$, counting multiplicities. The zeroes must all be at $z_{0}$; that is, $m-f$ has a zero of order at least 3 at $z_{0}$. This then implies $f^{\prime}\left(z_{0}\right)=m^{\prime}\left(z_{0}\right)$ and $f^{\prime \prime}\left(z_{0}\right)=m^{\prime \prime}\left(z_{0}\right)$, as needed.

Let $\varepsilon$ be the minimum of $|m(z)-f(z)|$ in the annulus $r / 2 \leqq\left|z-z_{0}\right| \leqq 2 r$. Let $n$ be sufficiently large so that $\left|m_{n}(z)-m(z)\right|<\varepsilon / 5$ and $\left|f_{n}(z)-f(z)\right|<\varepsilon / 5$ in this annulus, and the sizes of all the disks of $P^{n}$ meeting $\left\{z:\left|z-z_{0}\right| \leqq 3 r\right\}$, 
as well as the corresponding disks in $\widetilde{P}^{n}$, are very small. Let $\alpha^{*}$ be a simple closed path in $I^{n}$, which is a slight perturbation of $\alpha$, let $\gamma^{*}$ be the restriction of $m_{n}(z)-f_{n}(z)$ to $\alpha^{*}$, and let $A^{*}$ be the bounded component of $\mathbb{C}-\alpha^{*}$. It is not hard to see that $\gamma$ is homotopic to $\gamma^{*}$ in $\mathbb{C}-\{0\}$ (provided $\alpha^{*}$ is sufficiently close to $\alpha$, and $n$ sufficiently large), and hence their winding numbers are the same.

Let $J$ be the triangular interstice of $P^{n}$ that contains $w_{n}$, and let $a_{1}, a_{2}$ be two distinct points in $J$. Of course, we are assuming that $w_{n}$ is sufficiently close to $z_{0}$, so it is contained in $A^{*}$. In $J, m_{n}$ is equal to $g_{n}$, but $m_{n} \neq g_{n}$ at every point of $\alpha^{*}$. By the connectedness of $I^{n}$ there is some point $b \in I^{n} \cap A^{*}$, such that $m_{n}(b)=g_{n}(b)$ but there are points in $I^{n}$ arbitrarily close to $b$ where $m_{n} \neq g_{n}$. Take a point $b^{*}$ in $I^{n}$, very close to $b$, such that $m_{n}\left(b^{*}\right) \neq g_{n}\left(b^{*}\right)$, and let $\phi$ be the Möbius transformation that fixes $m_{n}\left(a_{1}\right)$, fixes $m_{n}\left(a_{2}\right)$ and takes $m_{n}\left(b^{*}\right)$ to $g_{n}\left(b^{*}\right)$. If $b^{*}$ is chosen sufficiently close to $b$, then $\phi$ is very close to the identity. Hence we assume that $\phi$ is sufficiently close to the identity so that the restriction of $\phi \circ m_{n}-g_{n}$ to $\alpha^{*}$ has the same winding number around 0 as $\gamma^{*}$. Set

$$
h=\phi \circ m_{n}-g_{n} .
$$

We also assume that $h$ has no zeroes in $\partial I^{n}$. There is no loss of generality in this assumption, because the set of choices of $b^{*}$ for which this would fail does not have interior. (Besides, we could also perturb $a_{1}$ and $a_{2}$.)

Now we are ready to estimate the winding number of the restriction of $h$ to $\alpha^{*}$, which is the same as the winding number of $\gamma^{*}$. In $A^{*} \cap I^{n}$ there are at least three zeroes of $h$, namely $a_{1}, a_{2}, b^{*}$. So the winding number of $h$ along $\partial\left(A^{*} \cap I^{n}\right)$ must be at least 3. Since the winding number of $h$ along $\partial P_{v}^{n}$ is nonnegative for each disk $P_{v}^{n}$ that intersects $A^{*}$ (by 3.1), and since the orientation of $\partial P_{v}^{n}$ is the opposite to its orientation as a part of $\partial I^{n}$, each such disk has a nonpositive contribution to the winding number of $h$ along $\partial\left(A^{*} \cap I^{n}\right)$. Hence, the winding number of $h$ along $\alpha^{*}=\partial A^{*}$ must be at least 3 , which completes the proof.

\section{Some generalizations}

We now note two generalizations of our work. First, instead of taking $P^{n}$ and $\widetilde{P}^{n}$ to be finite packings in $D$ and $\widetilde{D}$, respectively, it is natural to take infinite packings which completely fill out the domains, with nerve a triangulation of an open disk. The existence of such packings is proved in [11]. The Theorems 1.1 and 1.2 still hold in this situation. In fact, one can just apply the above theorems to large finite pieces of the packings.

The second generalization is not as trivial. It involves packings with nontriangular interstices.

6.1 Theorem Let $D, \widetilde{D} \varsubsetneqq \mathbb{C}$ be two domains, and assume that no component of $\partial D$ or of $\partial \widetilde{D}$ is a single point. Let $p_{0}$ be some point in $D$, and let $\delta_{n}$ be a sequence of positive numbers, tending to zero. For each $n$, let $\Omega^{n} \subset D$ be 
a compact set that contains $p_{0}$ and such that $\partial \Omega^{n}$ is contained in the spherical $\delta_{n}$ neighborhood of $\partial D$. Similarly, let $\widetilde{\Omega}^{n} \subset \widetilde{D}$ be compact with $\partial \Omega^{n}$ contained in the $\delta_{n}$ neighborhood of $\partial \widetilde{D}$. Let $P^{n}$ be a finite packing of disks in $\Omega^{n}$, and

let $\widetilde{P}^{n}$ be a finite packing of disks in $\widetilde{\Omega}^{n}$. Suppose that the spherical diameters of the disks in $P^{n}$ are at most $\delta_{n}$. Let $A^{n}$ be $\Omega^{n}$ minus the interior of $P^{n}$, and let $\widetilde{A}^{n}$ be $\widetilde{\Omega}^{n}$ minus the interior of $\widetilde{P}^{n}$.

Suppose that there is a homeomorphism $f_{n}: A^{n} \rightarrow \widetilde{A}^{n}$, which is conformal in the interior of $A^{n}$, and takes $\partial \Omega^{n}$ to $\partial \widetilde{\Omega}^{n}$. Further suppose that the set $\left\{f_{n}\left(p_{0}\right): n=1,2, \ldots\right\}$ has compact closure in $\widetilde{D}$. Then a subsequence of the sequence $f_{n}$ converges uniformly on compact subsets of $D$ to a conformal homeomorphism $f: D \rightarrow \widetilde{D}$. Moreover $f_{n}^{\prime} \rightarrow f^{\prime}$ and $f_{n}^{\prime \prime} \rightarrow f^{\prime \prime}$ uniformly on compact subsets of $D$.

Note that this theorem includes 1.1 and 1.2 as special cases.

The proof is essentially the same as the proofs above. One encounters only one sticky point, which comes up when proving the statements corresponding to Lemmas 2.1 and 2.2.

Consider, for example, Lemma 2.1. The problem is that the union $\widetilde{P}^{n}(\rho) \cup$ $\partial \operatorname{supp}\left(\widetilde{P}^{n}\right)$ does not have to separate $f_{n}\left(z_{0}\right)$ from $f_{n}\left(p_{0}\right)$. To overcome this, define $\widetilde{A}(\rho)$ to be the image under $f_{n}$ of $c_{s p}(\rho) \cap A^{n}$ and the boundaries of the circles of $P^{n}$ that intersect $c_{s p}(\rho)$. Then $\widetilde{A}(\rho) \cup \partial\left(\widetilde{P}^{n}\right)$ does separate $f_{n}\left(z_{0}\right)$ from $f_{n}\left(p_{0}\right)$.

Instead of estimating $L(\rho)$, which does not suffice now, one needs to estimate $L^{*}(\rho)$, which is the sum of the diameters of the images under $f_{n}$ of the circles of $P^{n}$ that intersect $c_{s p}(\rho)$ plus the sum of the lengths of the components of $f_{n}\left(c_{s p} \cap A^{n}\right)$. The first part of the sum is estimated just as in the original argument, while the second part is estimated by a continuous version of this. For examples of such mixed discrete and continuous estimates, see [21] or [10].

\section{References}

1. Beardon, A.F., Stephenson, K.: The Schwarz-Pick lemma for circle packings. Illinois J. Math. 141, 577-606 (1991)

2. Brightwell, G.R., Scheinerman, E.R.: Representations of planar graphs. SIAM J. Discrete Math. 6, 214-229 (1993)

3. Carleman, Comptes rendus 168, 843-845 (1919)

4. Colin De Verdiére, Y.: Un principe variationnel pour les empilements de cercles. Invent. math. 104, 655-669 (1991)

5. Doyle, P.G., He, Z.-X., Rodin, B.: Second derivatives of circle packings and conformal mappings. Discrete Comput. Geom. 11, 35-49 (1994)

6. Doyle, P.G., He, Z.-X., Rodin, B.: The asymptotic value of the circle-packing rigidity constants $s_{n}$. Discrete Comput. Geom. 12, 105-116 (1994)

7. He, Z.-X.: An estimate for hexagonal circle packings. J. Differ. Geom. 33, 395-412 (1991)

8. He, Z.-X., Rodin, B.: Convergence of circle packings of finite valence to Riemann mappings. Comm. Anal. Geom. 1, 31-41 (1993) 
9. He, Z.-X., Schramm, O.: Fixed points, Koebe uniformization and circle packings. Ann. Math. 137, 369-406 (1993)

10. He, Z.-X., Schramm, O.: Rigidity of circle domains whose boundary has $\sigma$-finite linear measure. Invent. Math. 115, 297-310 (1994)

11. He, Z.-X., Schramm, O.: Hyperbolic and parabolic packings. Discrete Comput. Geom. 14, 123-149 (1995)

12. Koebe, P.: Kontaktprobleme der konformen Abbildung. Berichte Verhande. Sächs: Akad. Wiss. Leipzig, Math.-Phys. Klasse 88, 141-164 (1936)

13. Marden, A., Rodin, B.: On Thurston's formulation and proof of Andreev's theorem. Lecture Notes in Mathematics 1435, 103-115, Springer, 1989

14. Rodin, B.: Schwarz's lemma for circle packings II. J. Differ. Geom. 30, 539-554 (1989)

15. Rodin, B., Sullivan, D.: The convergence of circle packings to the Riemann mapping. J. Differ. Geom. 26, 349-360 (1987)

16. Rudin, W.: Principles of Mathematical Analysis. 2nd edition, McGrow-Hill, New York, pp. 270, 1964

17. Schramm, O.: Packing two-dimensional bodies with prescribed combinatorics and applications to the construction of conformal and quasiconformal mappings. Ph.D. thesis, Princeton 1990

18. Schramm, O.: Existence and uniqueness of packings with specified combinatorics. Israel J. Math. 73, 321-341 (1991)

19. Schramm, O.: Rigidity of infinite (circle) packings. J. Am. Math. Soc. 4, 127-149 (1991)

20. Schramm, O.: Conformal uniformization and packings. Israel J. Math. 93, 399-428 (1996)

21. Schramm, O.: Transboundary extremal length. J. Anal. Math. 66, 307-329 (1995)

22. Stephenson, K.: Circle packings in the approximation of conformal mappings. Bull. Am. Math. Soc. 23, 407-415 (1990)

23. Thurston, W.P.: The finite Riemann mapping theorem, invited address, International Symposium in Celebration of the Proof of the Bieberbach Conjecture, Purdue University, 1985 